\title{
A NEW DISCRETE ANALOG OF THE LEGENDRE POLYNOMIALS
}

\author{
BY M. WAYNE WILSON
}

Communicated by Wallace Givens, October 2, 1969

1. Introduction. We introduce here a finite set of polynomials orthogonal over $N$ distinct points of $[-1,1]$ which are a very close analog of the Legendre polynomials. This set appears to have most of the properties of the Legendre (and ultraspheric) polynomials, yet they are not Fejér "generalized Legendre polynomials" (Szegö $[4, \S 6.5])$. These polynomials converge like $1 / N^{2}$ to the Legendre polynomials, in contrast to the Hahn polynomials $(\alpha=\beta=0)$ which converge like $1 / N$. (See Karlin and McGregor [2], Levit [3].) In every respect, they appear to be a superior analog of the Legendre polynomials than the Hahn polynomials (sometimes called the Gram or Chebyshev polynomials of discrete least squares).

2. The inner product. The Hahn polynomials arise naturally from equidistant point sets. In approximation theory, useful point sets are the zeros of the Chebyshev polynomials of the first and second kind $\left(T_{n}(x)\right.$ and $\left.U_{n}(x)\right)$. Let $\phi=\pi /(N+1)$, and $\psi=\frac{1}{2} \phi$. Let $t_{i}=\cos (i \phi)$, and $w_{i}=\sin (i \phi), i=1,2, \cdots, N$. Let $[,]_{N}$ be the inner product defined by $[f, g]_{N}=2 \tan \psi \sum_{i=1}^{N} w_{i} f\left(t_{i}\right) g\left(t_{i}\right)$, and let $(f, g)=f_{-1}^{1} f(t) g(t) d t$.

By means of the identity

$$
\begin{aligned}
\sum_{k=1}^{N} \sin k \alpha \phi & =\operatorname{cotan} \alpha \psi, & \alpha & =1,3,5, \cdots, \\
& =0, & \alpha & =0,2,4, \cdots
\end{aligned}
$$

one can show $[1,1]_{N}=2$, and that for fixed $n, N \geqq n \geqq 1,\left[t^{n}, t^{n}\right]_{N}$ monotonically increases (with $N$ ) to $\left(t^{n}, t^{n}\right)$, and that $\left[t^{n}, t^{n}\right]_{N}$ $=\left(t^{n}, t^{n}\right)+O\left(1 / N^{2}\right)$.

If we let $q_{k}(t ; N), k=0,1, \cdots, N-1$ be the monic form of the orthogonal polynomials of $[,]_{N}$, then, taking account of the symmetry of the inner product, the polynomials are given by the recurrence,

$q_{0} \equiv 1, \quad q_{1} \equiv t, \quad q_{n+1}=t q_{n}-\beta_{n}(N) q_{n-1}, \quad n=1,2, \cdots, N-2$, where $\beta_{n}(N)=\left[q_{n}, q_{n}\right]_{N} /\left[q_{n-1}, q_{n-1}\right]_{N}$. The polynomials $q_{n}(t ; N)$ are

AMS Subject Classifications. Primary 3340, 3327.

Key Words and Phrases. Discrete inner products, discrete orthogonal polynomials, orthogonal polynomials, Legendre polynomials. 
even or odd with $n$. There does not appear to be a simple closed form expression for $\beta_{n}(N)$. Applying Wilson [7], which shows how the convergence rate of $\left[1, t^{k}\right]$ affects other quantities of a discrete orthogonal system, we have $\beta_{n}(N)=n^{2} /\left(4 n^{2}-1\right)+O\left(N^{-2}\right)$. Computationally, it appears that $\beta_{n}(N)$ are monotonic increasing in $N$ for fixed $n$, and monotonic decreasing in $n$ for fixed $N$.

Letting $k_{n}$ be the leading coefficient of the Legendre polynomial $P_{n}(t)$, we have

$$
Q_{n}(t ; N) \equiv k_{n} q_{n}(t ; N)=P_{n}(t)+O\left(N^{-2}\right),
$$

using Wilson [7] again.

3. Expansion properties. One of the most remarkable features of these new polynomials is their expansion properties. Let $N \geqq n>0$, and suppressing $t$ and $N$ dependence, let

$$
\begin{aligned}
U_{n} & =A_{n}^{n} Q_{n}+A_{n-2}^{n} Q_{n-2}+A_{n-4}^{n} Q_{n-4}+\cdots \\
Q_{n} & =a_{n}^{n} U_{n}+a_{n-2}^{n} U_{n-2}+\cdots \\
Q_{n} & =P_{n}+B_{n-2}^{n} P_{n-2}+\cdots \\
Q_{n} & =C_{n}^{n} T_{n}+C_{n-2}^{n} T_{n-2}+\cdots \\
T_{n} & =c_{n}^{n} Q_{n}+c_{n-2}^{n} Q_{n-2}+\cdots
\end{aligned}
$$

By explicitly evaluating $\left[T_{n}, T_{m}\right]$ and $\left[T_{n}, U_{m}\right]$, using interrelations of the $T_{n}$ and $U_{n}$ polynomials, induction, and Wilson [5], we have shown that all the $A$ 's, $B^{\prime}$ s, $C^{\prime}$ 's are positive, $a_{n}^{n}$ and $c_{n}^{n}$ are positive for each $n$, and the remaining $a_{j}^{n}$ and $c_{j}^{n}$ are negative, $j=n-2$, $n-4, \cdots$.

These sign configurations are characteristic of the ultraspheric polynomials $P_{n}^{(\alpha, \alpha)}$, for $\alpha \in[-1 / 2,1 / 2]$. Loosely speaking, the $Q$ polynomials are "above" the Legendre polynomials $(\alpha=0)$, and "below" the second kind Chebyshev polynomials $(\alpha=1 / 2)$. Much other useful information is obtained in the course of finding these patterns, or is inferred by these expansions. Note that the $A_{n}^{n}, C_{n}^{n}$, $a_{n}^{n}, c_{n}^{n}$ coefficients are easily available explicitly. We have $B_{j}^{n}$ monotonically decreasing with $N$ to zero, with order $O\left(N^{-2}\right)$. This implies $Q_{n}(1, N)$ monotonically decreases to 1 , with order $O\left(N^{-2}\right)$. From (4), we have $\max \left\{\left|Q_{n}(t, N)\right| \mid t \in[-1,1]\right\}=Q_{n}(1, N)$. From (1), we can infer that the coefficients $C_{n}^{n}, C_{n-2}^{n}, \cdots$ monotonically decrease (for fixed $N$ ), and that $C_{2}^{n}>2 C_{0}^{n}$, for $n$ even. From this, it follows that if the zeros of $Q_{n}(t)$ are $\cos \theta_{\nu}^{(n)}, \nu=1,2, \cdots, n$, then 


$$
\frac{\nu-\frac{1}{2}}{n+1} \pi<\theta_{\nu}^{(n)}<\frac{\nu+\frac{1}{2}}{n+1} \pi, \quad \nu=1,2, \cdots, n
$$

by the same method as Szegö $[4, \S 6.5]$.

The monotonicity in (4), coupled with the formula

implies that

$$
C_{n}^{n}=\frac{2(2 n) !}{4^{n}(n !)^{2}}
$$

$$
Q_{n}(1 ; N)<\frac{2(2 n) !}{4^{n}(n !)^{2}}\left[\frac{n+1}{2}\right]
$$

where $[x]$ is the greatest integer containing $x$. Using Stirling's formula, this last quantity can be bounded by $(n+2) /(\pi n)^{1 / 2}$. For $n=29$, this expression is 3.25 , compared with $Q_{29}(1 ; 30)=2.42$.

4. General comments. The polynomial system $\left\{Q_{n}(t ; N)\right\}_{n=0}^{N-1}$ appears to share most of the nice properties of the Legendre polynomials, in complete contrast to the Hahn polynomials, which look like the Legendre polynomials only for $N$, the number of points, of the order of $n^{2}$. See Wilson [6] for some details. Numerical evidence suggests that $Q_{n}(t ; N) \cdot Q_{m}(t ; N)$ has a nonnegative expansion in the $Q$ system polynomials, $n+m<N$. Again from computational evidence, the maxima of $\left|Q_{n}(t ; N)\right|$ seem to decrease as $t$ moves from -1 to 0 , or +1 to 0 , like the Legendre polynomials.

For finite $N, N>n, n \geqq 4$, we can prove that the sequence $Q_{0}, Q_{1}$, $\cdots, Q_{n}$ is not proportional to a set of "Fejér generalized Legendre" polynomials; consequently, the polynomials are also not proportional to ultraspherical polynomials. Further, the $O\left(\mathrm{~N}^{-2}\right)$ convergence shows that the polynomials are not Hahn polynomials. The work of Wynn [9] and Hahn [1] then shows that there is no difference analog of "Rodrigues' formula" for these polynomials.

From an approximation viewpoint, numerical experimentation shows that for continuous functions $f(t)$ on $[-1,1]$, the Fourier coefficients $\left[f, Q_{k}\right]_{N} /\left[Q_{k}, Q_{k}\right]_{N}$ are much closer to the LegendreFourier coefficient $\left(f, P_{k}\right) /\left(P_{k}, P_{k}\right)$ than the corresponding coefficient for the Hahn or usual least squares polynomial (Wilson [8]).

Finally, a word about motivation. Consider the inner product

$$
[f, g]_{N}=\sum_{i=0}^{N+1} \prime \prime\left(w_{i}\right)^{1+2 \alpha} f\left(t_{i}\right) g\left(t_{i}\right)
$$

where the primes indicate that the first and last terms are weighted by $1 / 2$. (We define $(0)^{0} \equiv 1$.) For $\alpha=+1 / 2, \alpha=-1 / 2$, it is well known 
that the discrete orthogonal polynomials that are generated are respectively $U_{n}(t)$ and $T_{n}(t)$. These are proportional to the Ultraspherical polynomials $P_{n}^{(1 / 2,1 / 2)}$ and $P_{n}^{(-1 / 2,-1 / 2)}$.

Since the Legendre polynomials are $P_{n}^{(0,0)}$, it is natural to try $\alpha=0$ in the above inner product. Riemann sum considerations show that for general $\alpha$, the inner product will generate analogs of the ultraspherics. An open question is, what is the order of convergence?

\section{REFERENCES}

1. W. C. Hahn, Über Orthogalpolynome, die q-Differenzgleichungen genügen, Math. Nachr. 2 (1949), 4-34. MR 11, 29.

2. S. Karlin and J. L. McGregor, The Hahn polynomials, formulas, and an application, Scripta Math. 26 (1961), 33-46. MR 25 \#2249; MR 30 p. 1202.

3. R. J. Levit, The zeros of the Hahn polynomials, SIAM Rev. 9 (1967), 191-203. MR $35 \# 4485$.

4. G. Szegö, Orthogonal polynomials, 2nd rev. ed., Amer. Math. Soc. Colloq, Publ., vol. 23, Amer. Math. Soc., Providence, R. I., 1959. MR 1, 14; MR 21 \#5029.

5. M. W. Wilson, Nonnegative expansions of polynomials, Proc. Amer. Math. Soc. 24 (1970), 100-102.

6. - On the Hahn polynomials, SIAM J. Math. Anal. 1 (1970), 37-43.

7. - Convergence properties of discrete analogs of orthogonal polynomials, Computing J. (to appear).

8. - Two discrete analogs of Legendre polynomials, a comparison, RC 2318, IBM Watson Research Center, Yorktown Heights, New York, 1968.

9. P. Wynn, A general system of orthogonal polynomials, Quart. J. Math. Oxford (2), 18 (1967), 81-96. MR $35 \# 1848$.

IBM Watson Research Center, Yorktown Heights, New York 10598 